\title{
Free group automorphisms, invariant orderings and topological applications
}

\author{
Dale Rolfsen \\ BERT WIEST
}

\begin{abstract}
We are concerned with orderable groups and particularly those with orderings invariant not only under multiplication, but also under a given automorphism or family of automorphisms. Several applications to topology are given: we prove that the fundamental groups of hyperbolic nonorientable surfaces, and the groups of certain fibred knots are biorderable. Moreover, we show that the pure braid groups associated with hyperbolic nonorientable surfaces are left-orderable.
\end{abstract}

\section{AMS Classification 6F15; 57M05}

Keywords Ordered group, surface group, knot group, surface braid group

\section{Introduction}

If $G$ is a group, and $<$ a strict total ordering of its elements, we say that $(G,<)$ is a left-ordered group if $x<y \Leftrightarrow z x<z y$ for all $x, y, z \in G$, and bi-ordered if the ordering is also right-invariant: $x<y \Leftrightarrow x z<y z$. Surprisingly many groups are left-orderable or even bi-orderable; for example free groups are biorderable [3], although this is by no means obvious (one method is described in Section 3).

If $\varphi: G \rightarrow G$ is an automorphism, then we say that an ordering $<$ of $G$ is invariant under $\varphi$ (or respected by $\varphi$ ) if $x<y \Leftrightarrow \varphi(x)<\varphi(y)$ for all $x, y \in G$. As we will see, it may or may not be possible to find such an invariant ordering, depending on the nature of the $\varphi$. In fact all our results on such invariant orderings involve free groups.

The goal of this paper is to establish new orderability results for several families of groups which arise in topology. We use a sort of bootstrap process involving extensions and orderings of free subgroups which also invariant under certain automorphisms. We show the following results: 
(1) The fundamental groups of all closed surfaces, orientable or not, are biorderable, with the exceptions of the projective plane and the Klein bottle (cf. [4]).

(2) The pure braid groups $P B_{n}(N)$ associated with nonorientable surfaces $N \neq \mathbb{P}^{2}$ are left-orderable.

(3) The fundamental groups of certain punctured-torus bundles over the circle are bi-orderable; examples include the figure-of-eight knot group.

Some of our technical arguments are directly adapted from [5] (which in turn built on ideas from [6]). In these papers it was proved that the pure braid groups, and more generally all pure surface braid groups (in any orientable compact surface) are bi-orderable. Moreover, [5] shows that $P B_{n}(N)$ is not bi-orderable for nonorientable surfaces $N$.

More general surveys on orderable groups and their rôle in low-dimensional topology, which place our results in their proper context, can be found in the introductory sections of [4] and [12].

We thank Steven Boyer for many helpful discussions; some of the present results arose directly from our work on the orderability of 3-manifold groups. Thanks also to Juan Gonzales-Meneses for inspiring discussions. Dale Rolfsen was partially supported by research grant of the Canadian Natural Sciences and Engineering Research Council, and Bert Wiest by a PIMS postdoctoral fellowship.

\section{Orderable groups and extensions}

Groups which are left-orderable are easily seen to be torsion-free. However, left-orderable groups enjoy certain advantages over merely torsion-free ones; for instance, they are known to satisfy the (still open) zero-divisor conjecture, which states that the group ring $\mathbb{Z} G$ of a torsion free group $G$ should have no zero-divisors. The group rings of bi-orderable groups are known to have an even stronger property, due to Malcev and Neumann (and conjectured to be true for left-orderable groups as well): if $G$ is a bi-orderable group, then $\mathbb{Z} G$ embeds in a division algebra. We refer the reader to [3] for proofs and more general statements.

It is easy to verify that a group $G$ is left-orderable if and only if there is a subset $P \subset G$ which does not contain the identity element, is closed under multiplication and such that for every $g \neq 1$, exactly one of $g$ and $g^{-1}$ belongs 
to $P$. Given such a $P$, define $g<h \Leftrightarrow g^{-1} h \in P$. On the other hand, given a left-ordering $<$, define $P$ to be the positive cone $P=\{g \in G: 1<g\}$. Note that if one instead used the criterion $g<h \Leftrightarrow h g^{-1} \in P$, a right-invariant ordering would result: a group is right-orderable if and only if it is left-orderable.

A left-ordering is bi-invariant if and only if its positive cone $P$ is normal: $g^{-1} P g \subset P \quad \forall g \in G$. Moreover, the ordering is invariant under an automorphism $\varphi$ if and only $\varphi(P) \subset P$. Following is one of the reasons to be interested in orderings invariant under automorphisms. Its straightforward proof is left to the reader.

Lemma 1 Suppose we have a short exact sequence of groups

$$
1 \longrightarrow F \hookrightarrow G \stackrel{p}{\longrightarrow} H:=G / F \longrightarrow 1 \text {. }
$$

If $F$ and $H$ are left-orderable, then $G$ is left-orderable, with positive cone $P_{G}:=p^{-1}\left(P_{H}\right) \cup P_{F}$. If $F$ and $H$ are bi-ordered, then the same formula defines the positive cone for a bi-ordering of $G$ if and only if $g^{-1} P_{F} g \subset P_{F}$ for all $g \in G$, that is, if and only if the ordering of $F$ is invariant under conjugation by elements of $G$.

This gives us a strategy to prove that a group $G$ is bi-orderable. One finds a convenient normal subgroup $F$ such that $G / F$ is bi-orderable and in addition $F$ can be given a bi-ordering invariant under conjugation by elements of $G$.

\section{Surface groups}

It has been known for a long time $[1,8]$ that the fundamental groups of orientable surfaces are bi-orderable (and in particular left-orderable - an interesting non-constructive argument in [2] also shows this). Our aim is to generalize these results to non-orientable surfaces, using very different techniques.

We shall denote the connected sum of $n$ projective planes by $n \mathbb{P}^{2}$. We recall that $(n+2) \mathbb{P}^{2}$ is homeomorphic to the connected sum of a torus and $n$ projective planes. Consider $3 \mathbb{P}^{2} \cong T^{2} \# \mathbb{P}^{2}$, the nonorientable surface with Euler characteristic -1 ; this surface will be the key to our analysis. Note that $n \mathbb{P}^{2}$ has a hyperbolic structure for $n \geqslant 3$, whereas $\mathbb{P}^{2}$ is spherical and the Klein bottle $2 \mathbb{P}^{2}$ is Euclidean.

Proposition 2 The group $\pi_{1}\left(3 \mathbb{P}^{2}\right)$ is bi-orderable. 
Theorem 3 If $N$ is any connected surface other than the projective plane $\mathbb{P}^{2}$ or Klein bottle $2 \mathbb{P}^{2}$, then $\pi_{1}(N)$ is bi-orderable. For $N=$ Klein bottle, $\pi_{1}(N)$ is left-orderable.

Proof of theorem 3 Let us first see how theorem 3 follows from proposition 2. If $N$ is noncompact, or if $\partial N$ is nonempty, then $\pi_{1}(N)$ is a free group, and therefore bi-orderable. Thus we are reduced to considering closed surfaces. According to the standard classification, such surfaces are either a connected sum of tori, or of projective planes in the nonorientable case.

We shall first consider non-orientable surfaces. Of course $\pi_{1}\left(\mathbb{P}^{2}\right) \cong \mathbb{Z}_{2}$ is certainly not left-orderable. For $N=2 \mathbb{P}^{2}$, the Klein bottle,

$$
\pi_{1}(N) \cong\left\langle x, y: x y x^{-1}=y^{-1}\right\rangle
$$

is a well-known example of a group which is left-orderable (being an extension of $\mathbb{Z}$ by $\mathbb{Z}$ ), but not bi-orderable, as the defining relation would lead to a contradiction.

By proposition 2 , the surface $M=3 \mathbb{P}^{2} \cong T^{2} \# \mathbb{P}^{2}$ has bi-orderable fundamental group. We shall picture it as a torus with a small disk removed, and replaced by sewing in a Möbius band. Consider an $n$-fold cover of the torus by itself, and modify the covering by replacing one disk downstairs, and $n$ disks upstairs, by Möbius bands. This gives a covering of $M$ by the connected sum of a torus with $n$ copies of $\mathbb{P}^{2}$. Thus the fundamental group of $(n+2) \mathbb{P}^{2}$ injects in that of $3 \mathbb{P}^{2}$, and is therefore bi-ordered.

We now turn to orientable closed surfaces. The cases of genus zero or one being easy, we consider a closed surface of genus $g \geqslant 2$. This surface is the oriented double cover of $(g+1) \mathbb{P}^{2}$. Therefore its fundamental group is a subgroup of a biorderable group. This completes the proof of theorem 3, assuming proposition 2 .

Corollary 4 The pure braid group $P B_{m}(N)$ on $m$ strands in a compact surface $N \neq \mathbb{P}^{2}$ is left-orderable.

If $N$ is non-orientable, this is the strongest possible result, because by [5] the groups $P B_{m}(N)$ are definitely not bi-orderable. If $N$ is orientable, the result is redundant, since in this case $P B_{m}(N)$ is known to be even bi-orderable [5]. We also remark that it is not known which non-pure surface braid groups are left-orderable. 
Proof of corollary 4 We shall proceed by induction on $m$. For $m=1$, we have that $P B_{1}(N)=\pi_{1}(N)$ is left-orderable by theorem 3. Moreover, we have a short exact sequence

$$
1 \longrightarrow \pi_{1}(N-\{m \text { points }\}) \hookrightarrow P B_{m+1}(N) \stackrel{\phi}{\longrightarrow} P B_{m}(N) \longrightarrow 1,
$$

where $\phi$ is induced by forgetting one of the strands. Now $\pi_{1}(N-\{m$ points $\})$ is free and hence left-orderable, and $P B_{m}(N)$ is left-orderable by induction; an application of lemma 1 completes the induction step.

Proof of proposition 2 Let $M=3 \mathbb{P}^{2} \cong T^{2} \# \mathbb{P}^{2}$. Our strategy for constructing a bi-invariant order on $\pi_{1}(M)$ is to apply lemma 1 , where the normal subgroup $F$ of $G=\pi_{1}(M)$ will be chosen so that $G / F \cong \mathbb{Z}^{2}$.

To define the subgroup $F$, we note that $G$ has presentation

$$
G=\left\langle a, b, c: a b a^{-1} b^{-1}=c^{2}\right\rangle
$$

where $a$ and $b$ represent free generators of the punctured torus in $M$ and $c$ the generator corresponding to the central curve of the Möbius band in $M$. We define $F=\langle\langle c\rangle\rangle$, the normal subgroup generated by $c$. Note that a word in $a, b, c$ belongs to $F$ if and only its exponent sums in $a$ and $b$ are both zero.

The covering $\widetilde{M}$ of $M$ with $\pi_{1}(\widetilde{M})=F$ is very easy to imagine: consider the universal covering $\mathbb{R}^{2} \rightarrow T^{2}$, and modify $\mathbb{R}^{2}$ by taking a family of small disks (say $\left.D_{i, j}\right)$ centered at the integral points $(i, j) \in \mathbb{Z}^{2}$. Remove each of these and replace by a Möbius band $B_{i, j}$. This defines a covering $\widetilde{M} \rightarrow M$. The group of covering translations of $\widetilde{M}$ is just $\mathbb{Z}^{2}$, with $(m, n)$ acting by translation $(x, y) \rightarrow(x+m, y+n)$ on the $\mathbb{R}^{2}$ part of $\widetilde{M}$, and taking each $B_{i, j}$ to $B_{i+m, j+n}$. Therefore we have (as required) an exact sequence

$$
1 \rightarrow \pi_{1}(\widetilde{M}) \rightarrow \pi_{1}(M) \rightarrow \mathbb{Z}^{2} \rightarrow 1 .
$$

We now turn to the task of proving that the orderability hypotheses of lemma 1 are satisfied. The group $\mathbb{Z}^{2}$ can be bi-ordered, say lexicographically. (In fact, there are uncountably many different bi-orders on $\mathbb{Z}^{2}$; e.g. there are already two for each line of irrational slope in $\mathbb{R}^{2}$ through $(0,0)$.)

All that remains to be proven is that $F \cong \pi_{1}(\widetilde{M})$ has a bi-ordering which is invariant under conjugation by elements in $G=\pi_{1}(M)$. We note that $F$ is an infinitely-generated free group. There is a free basis for $F$ consisting of the generators $x_{i, j}$ represented by a loop that goes around the central curve of the Möbius band $B_{i, j}$, connected by a tail to the basepoint in some (non-canonical) way; for definiteness we may take

$$
x_{i, j}=a^{i} b^{j} c b^{-j} a^{-i} \quad\left((i, j) \in \mathbb{Z}^{2}\right)
$$


as a free generating set for $F$. Now $G$ acts upon $F$ by conjugation, which may be described in terms of the generators as follows.

Lemma 5 Suppose $g \in G$ has exponent sums $m$ and $n$ in $a$ and $b$, respectively. Then

$$
g x_{i, j} g^{-1}=w_{i, j} x_{i+m, j+n} w_{i, j}^{-1},
$$

where $w_{i, j}=w_{i, j}(g) \in F$.

Proof of the lemma We just take $w_{i, j}=g a^{i} b^{-n} a^{-i-m}$; by calculating the exponent sums of $a$ and $b$ in $w_{i, j}$ we can verify that indeed $w_{i, j} \in F$.

For the following, $F_{a b}$ denotes the abelianization of $F$, which is an infinitely generated free abelian group, with generators, say $\widetilde{x}_{i, j}$; the abelianization map $F \rightarrow F_{a b}$ is just $x_{i, j} \rightarrow \widetilde{x}_{i, j}$. Any automorphism $\phi$ of $F$ induces a unique automorphism $\phi_{a b}$ of $F_{a b}$. For example, in the above lemma, under abelianization the conjugation map is just the shift $\widetilde{x}_{i, j} \rightarrow \widetilde{x}_{i+m, j+n}$. Now lemma 6 completes the proof of proposition 2 .

Lemma 6 There is a bi-ordering of the free group $F=\left\langle x_{i, j}\right\rangle ;(i, j) \in \mathbb{Z}^{2}$ which is invariant under every automorphism $F \rightarrow F$ which induces, on $F_{a b}$, a uniform shift automorphism $\widetilde{x}_{i, j} \rightarrow \widetilde{x}_{i+m, j+n}$.

Proof We use the Magnus expansion [9], sending $F$ into the ring of formal power series in the infinitely many noncommuting variables $X_{i, j}$. Since there are infinitely many generators, some care must be taken in defining the ring $\mathbb{Z}\left[\left[X_{i, j}\right]\right]$, which we take to be the ring consisting of formal power series in the $X_{i, j}$, but we consider only such series which involve just finitely many different variables. The Magnus map $\mu: F \rightarrow \mathbb{Z}\left[\left[X_{i, j}\right]\right]$ is given by

$$
\mu\left(x_{i, j}\right)=1+X_{i, j} ; \quad \mu\left(x_{i, j}^{-1}\right)=1-X_{i, j}+X_{i, j}^{2}-X_{i, j}^{3}+\cdots
$$

Clearly the image of $F$ lies in the group of units of the form $\{1+O(1)\}$ inside $\mathbb{Z}\left[\left[X_{i, j}\right]\right]$, and it is an embedding of groups, by the same proof as in [9]. Elements of $\mathbb{Z}\left[\left[X_{i, j}\right]\right]$ may be written in standard form, arranged in ascending degree, and within a degree terms are arranged lexicographically by their subscripts (which in turn are ordered lexicographically). Then two series are compared according to the coefficient of the first term at which they differ (here is where the finiteness assumption is necessary). The proof that this defines a (multiplicative) bi-invariant ordering of $\{1+O(1)\}$, is routine - cf. [6]. Via the injection $\mu$, we may regard $F$ as a subgroup, and hence it is also biordered. 
Finally, we argue that this ordering has the desired invariance property; equivalently, that $\phi$ preserves the positive cone of $F$. Consider an automorphism $\phi: F \rightarrow F$ such that $\phi_{a b}: F_{a b} \rightarrow F_{a b}$ is a shift $\phi_{a b}\left(\widetilde{x}_{i, j}\right)=\widetilde{x}_{i+m, j+n}$. This means that $\phi\left(x_{i, j}\right)=x_{i+m, j+n} c_{i, j}$, where $c_{i, j}$ is in the commutator subgroup $[F, F]$. Since $[F, F]$ maps into $\{1+O(2)\}$ under the Magnus embedding, the effect of $\phi$ is reflected in $\mathbb{Z}\left[\left[X_{i, j}\right]\right]$ by the substitution $X_{i, j} \rightarrow X_{i+m, j+n}+O(2)$. Therefore, if the Magnus expansion of $w \in F$ is $\mu(w)=1+w_{d}\left(X_{i, j}\right)+O(d+1)$, where $w_{d}$ is the sum of all degree $d$ terms, then $\mu \phi(w)=1+w_{d}\left(X_{i+m, j+n}\right)+$ terms of higher degree. Therefore, the lowest degree nonzero terms of the Magnus expansions of $w$ and $\phi(w)$ are identical, except that the subscripts are shifted. Thus the "first" nonconstant terms of both $w$ and $\phi(w)$ have the same coefficient, and we conclude that $\phi$ preserves the positive cone of $F$ in the ordering we described.

\section{Punctured-torus bundles over the circle}

Suppose that a 3-manifold $M^{3}$ is a fibre bundle over the circle, with fibre some surface $S$, and monodromy map $\varphi: S \rightarrow S$. A necessary and sufficient condition for $\pi_{1}\left(M^{3}\right)$ to be bi-ordered is that there exists some bi-order on $\pi_{1}(S)$ which is invariant under $\varphi$. This can be seen by considering the short exact sequence of the fibration

$$
1 \longrightarrow \pi_{1}(S) \hookrightarrow \pi_{1}\left(M^{3}\right) \longrightarrow \mathbb{Z} \longrightarrow 1
$$

and applying lemma 1 . It is an important and apparently hard problem in general to decide which automorphisms of free groups or surface groups leave some bi-ordering invariant.

In the present paper we restrict our attention to the case where $S$ is a oncepunctured torus - for a different line of attack see [11]. We shall apply a slightly more sophisticated version of lemma 6 in order to prove

Theorem 7 Suppose the 3-manifold $M^{3}$ is a fibre bundle over the circle, with fibre a once-punctured torus $S$. Suppose that the monodromy map $\varphi: S \rightarrow S$ is orientation preserving and induces in homology a homomorphism $\Phi: H_{1}(S) \rightarrow$ $H_{1}(S)$ which preserves some bi-ordering on $H_{1}(S) \cong \mathbb{Z}^{2}$. Then $\pi_{1}\left(M^{3}\right)$ is bi-orderable.

It is known which automorphisms of $\mathbb{Z}^{n}$ leave some bi-order invariant. This is due to Levitt [7]: suppose $T: \mathbb{C}^{n} \rightarrow \mathbb{C}^{n}$ is a linear transformation that restricts to an automorphism $T: \mathbb{Z}^{n} \rightarrow \mathbb{Z}^{n}$ (i.e. it is represented by some $n \times n$ matrix 
with integer entries, and determinant \pm 1 ). Consider a basis $\mathcal{B}$ of $\mathbb{C}^{n}$ such that the matrix of $T$ with respect to $\mathcal{B}$ is in Jordan normal form. We can split $\mathbb{C}^{n}$ uniquely as a direct sum $E \oplus F$, where we define $E$ to be spanned by the vectors of $\mathcal{B}$ belonging to Jordan blocks with positive real eigenvalue, and $F$ to be spanned by the vectors of $\mathcal{B}$ with negative or complex eigenvalues. Now Levitt's criterion is: the homomorphims $T: \mathbb{Z}^{n} \rightarrow \mathbb{Z}^{n}$ leaves some biorder on $\mathbb{Z}^{n}$ invariant if and only if $F$ does not intersect the integer lattice $\mathbb{Z}^{n} \subseteq \mathbb{C}^{n}$ except in 0 . The proof is not difficult, and left to the reader - the basic observation is that for any bi-ordering of $\mathbb{Z}^{n}$ there exists a hyperplane in $\mathbb{R}^{n}$ such that all integer lattice points to one side of the plane are in the positive, and those on the other side in the negative cone.

We remark that if the monodromy map is orientation reversing, then $\pi_{1}\left(M^{3}\right)$ is definitely not bi-orderable, because it contains a Klein bottle group. Also, if the monodromy map is periodic, then $\pi_{1}\left(M^{3}\right)$ cannot be bi-orderable, because a periodic automorphism of $\pi_{1}(S)$ cannot leave a bi-ordering of $\pi_{1}(S)$ invariant. This proves, for example, that the group of the trefoil knot, which is a fibred knot with a monodromy map of period 6 , is not bi-orderable. This fact, which was pointed out in [10], seems to have dissuaded mathematicians from studying bi-orderability of knot groups for the last 25 years.

Examples for theorem 7 An orientation preserving (i.e. determinant 1) automorphism $\Phi$ of $\mathbb{Z}^{2}$ preserves a bi-order if and only if its eigenvalues are both positive (but not necessarily distinct). For if $\Phi$ has eigenvalues $\lambda_{1}, 1 / \lambda_{1}>0$, where $\overrightarrow{v_{1}}$ is the eigenvector for $\lambda_{1}$ and $\overrightarrow{v_{2}}$ is the other basis vector in a Jordan normal basis, then we can define the positive cone of a bi-ordering of $\mathbb{Z}^{2}$ by

$$
P_{\mathbb{Z}^{2}}=\left\{\vec{z}=c_{1} \overrightarrow{v_{1}}+c_{2} \overrightarrow{v_{2}} \in \mathbb{Z}^{2}: c_{2}>0 \text { or }\left(c_{2}=0 \text { and } c_{1}>0\right)\right\} .
$$

We observe that this order is invariant under $\Phi$.

For instance, theorem 7 implies that the complement of the figure-of-eight knot has bi-orderable fundamental group, because it is a fibred knot with punctured torus fibre $S$, and the matrix of the monodromy action on $H_{1}(S)$ is $\left(\begin{array}{ll}2 & 1 \\ 1 & 1\end{array}\right)$, which has two positive eigenvalues. The knot $4_{1}$ is the only classical knot (in $S^{3}$ ) which is covered by theorem 7 , because the only classical fibred knots of genus 1 are the knots $3_{1}$ and $4_{1}$. A few more classical knots are bi-orderable by the main theorem of [11].

If, on the other hand, $\Phi$ has two negative, or two complex conjugate eigenvalues, then it cannot respect any decomposition of $\mathbb{Z}^{2}$ into a "positive" and a "negative" half-space; thus $\Phi$ cannot preserve any ordering on $\mathbb{Z}^{2}$. 
Proof of theorem 7 Consider the short exact sequence

$$
1 \longrightarrow \pi_{1}(\widetilde{S}) \longrightarrow \pi_{1}(S) \stackrel{a b}{\longrightarrow} H_{1}(S) \longrightarrow 1,
$$

where $a b$ is the abelianisation homomorphism (which geometrically corresponds to "patching the puncture in $S$ "), and $\widetilde{S}$ is the covering space of $S$ whose fundamental group is the commutator subgroup $\left[\pi_{1}(S), \pi_{1}(S)\right]$. One can picture $\widetilde{S}$ as the plane $\mathbb{R}^{2}$ with all integer lattice points $\mathbb{Z}^{2}$ removed.

In particular, $\pi_{1}(\widetilde{S})$ is an infinitely generated free group, with generating set $\left\{x_{i, j} \mid(i, j) \in \mathbb{Z}^{2}\right\}$, where the loop $x_{i, j}$ has winding number one around the puncture at the point $(i, j) \in \mathbb{R}^{2}$, and winding number zero around the punctures at all the other integer lattice points (many different choices are possible here). Note that the abelianisation $H_{1}(\widetilde{S})$ is an infinitely generated free abelian group with generators $\left\{\widetilde{x}_{i, j}:(i, j) \in \mathbb{Z}^{2}\right\}$.

We have to prove that $\phi_{*}: \pi_{1}(S) \rightarrow \pi_{1}(S)$ leaves some bi-order on $\pi_{1}(S)$ invariant. We already have a $\Phi$-invariant bi-order on $H_{1}(S)$, and in fact we shall take it to be the order constructed in the example for theorem 7 . Thus it suffices by lemma 1 to find a bi-order on $\pi_{1}(\widetilde{S})$ which is

(i) invariant under conjugation by elements in $\pi_{1}(S)$, and

(ii) invariant under (the restriction of) $\phi_{*}$.

Let's study the effect of conjugation by elements in $\pi_{1}(S)$ and of $\phi_{*}$. Conjugation by an element $g \in \pi_{1}(S)$ with $a b(g)=(n, m) \in \mathbb{Z}^{2}$ sends a generator $x_{i, j}$ to $w_{i, j} x_{i+m, j+n} w_{i, j}^{-1}$, where $w_{i, j} \in \pi_{1}(\widetilde{S})$ depends on $g$, as in lemma 5 . Thus, on the abelianisation $H_{1}(\widetilde{S})$, conjugation by $g$ induces a uniform shift automorphism $\widetilde{x}_{i, j} \rightarrow \widetilde{x}_{i+m, j+n}$. (Note that the mapping $\mathbb{Z}^{2} \rightarrow \mathbb{Z}^{2},(i, j) \mapsto(i+m, j+n)$ preserves our ordering of $\mathbb{Z}^{2}$.)

Moreover, the restriction of $\phi_{*}$ to $\pi_{1}(\widetilde{S})$ is given geometrically by the action of the lift $\widetilde{\phi}: \widetilde{S} \rightarrow \widetilde{S}$ which fixes the point $(0,0)$. This sends the generator $x_{i, j}$ to a conjugate of the generator $x_{\Phi(i, j)}$, and induces on the abelianisation $H_{1}(\widetilde{S})$ an automorphism determinded by another simple permutation of the generators: $\widetilde{x}_{i, j} \mapsto \widetilde{x}_{\Phi(i, j)}$. The proof of theorem 7 is now completed by the following

Lemma 8 There is a bi-ordering of the free group $\pi_{1}(\widetilde{S})=\left\langle x_{i, j}\right\rangle ;(i, j) \in \mathbb{Z}^{2}$ which is invariant under every automorphism which induces, on the abelianisation $H_{1}(\widetilde{S})$, an automorphism that acts simply by permuting the variables: $\widetilde{x}_{i, j} \rightarrow \widetilde{x}_{\sigma(i, j)}$, where the permutation $\sigma: \mathbb{Z}^{2} \rightarrow \mathbb{Z}^{2}$ preserves the bi-ordering of $\mathbb{Z}^{2}$. 
Proof The proof is virtually the same as for lemma 6 . We order the variables $\left\{X_{i, j}\right\}$ by defining $X_{i, j}$ to "come before" $X_{i^{\prime}, j^{\prime}}$ if $(i, j)>\left(i^{\prime}, j^{\prime}\right)$ in the $\Phi$ invariant bi-order of $\mathbb{Z}^{2}$. Then in order to define which of two words in $\pi_{1}(\widetilde{S})$ is the larger we compare their images under the Magnus map $\mu$. Finally the invariance property is proved precisely as in lemma 6 .

\section{References}

[1] G. Baumslag, On generalised free products, Math. Z. 78 (1962), 423 - 438

[2] B. H. Bowditch, A variation on the unique product property, J. London Math. Soc. (2) 62 (2000), 813-826.

[3] R. Botto Mura, A. H. Rhemtulla, Orderable groups. Lecture Notes in Pure and Applied Mathematics, Vol. 27. Marcel Dekker Inc., New York-Basel, 1977

[4] S. Boyer, D. Rolfsen, B. Wiest, Orderable 3-manifold groups, preprint

[5] J. Gonzalez-Meneses, Ordering pure braid groups on closed surfaces, to appear in Pacific J. Math.

[6] D. Kim, D. Rolfsen, Ordering groups of pure braids and hyperplane arrangements, UBC preprint, 1999

[7] G. Levitt, private communication

[8] D. Long, Planar kernels in surface groups, Quart. J. Math. Oxford (2), 35 (1984), $305-310$

[9] W. Magnus, A. Karass, D. Solitar, Combinatorial group theory, Pure and Applied Math. Vol. XIII, Interscience Publishers, 1966

[10] L. Neuwirth, The status of some problems related to knot groups. (Topology Conference, Virginia Polytech. Inst. and State Univ., Blacksburg, Va., 1973), 209-230. Lecture Notes in Math., Vol. 375, Springer, Berlin, 1974

[11] B. Perron, D. Rolfsen, Ordering certain fibred knot groups, preprint.

[12] H. Short, B. Wiest, Orderings of mapping class groups after Thurston, L'enseignement mathématique 46 (2000), 279-312

Mathematics Department, University of British Columbia, 1984 Mathematics Road, Vancouver BC, Canada V6T $1 Z 2$

Email: rolfsen@math.ubc.ca, bertw@pims.math.ca

Received: 8 February $2001 \quad$ Revised: 16 May 2001 